\title{
ÉPOCA Y DENSIDAD DE SIEMBRA EN LA PRODUCCIÓN Y CALIDAD DE SEMILLA DE TRITICALE (X Triticosecale Wittmack) ${ }^{1}$
}

\author{
Mariano Mendoza-Elos ${ }^{2}$,Elvira Cortez-Baheza ${ }^{2}$, José Guadalupe Rivera-Reyes ${ }^{2}$, José Antonio Rangel-Lucio ${ }^{2}$, \\ Enrique Andrio-Enríquez $z^{2}$,Francisco Cervantes-Ortiz ${ }^{2}$
}

\section{RESUMEN}

Época y densidad de siembra en la producción y calidad de semilla de triticale (X Triticosecale Wittmack). El objetivo del presente trabajo fue determinar el efecto de la densidad y la fecha de siembra en el rendimiento y calidad de semilla de triticale. El ensayo se realizó durante el ciclo otoño-invierno, 2006-2007, en el campo experimental del Instituto Tecnológico de Roque, ubicado en el Km. 8 de la carretera Celaya-Juventino Rosas, en Celaya, Guanajuato, México. Se utilizaron tres cultivares de triticale de diferente origen genético (Roque I, AN-31 y AN-105) con una fórmula por hectárea de 120-100-100, NPK. Se evaluaron tres fechas de siembra: nueve de noviembre, veintitrés de noviembre, y el siete de diciembre. En cada fecha se sembraron cinco densidades de siembra, $50 \mathrm{~kg} / \mathrm{ha}, 75 \mathrm{~kg} / \mathrm{ha}, 100 \mathrm{~kg} / \mathrm{ha}, 125$ $\mathrm{kg} / \mathrm{ha}$ y $150 \mathrm{~kg} / \mathrm{ha}$. Las variables medidas fueron altura de la planta, longitud de la espiga, rendimiento de semilla, vigor y germinación. Se empleó un diseño de bloques completos al azar con arreglo factorial. La siembra realizada el día 9 de noviembre mostró el mayor rendimiento (5305 t/ha); y mayores valores de longitud de espiga, altura de planta, vigor y germinación. Los mejores resultados se obtuvieron cuando se establecieron $125 \mathrm{~kg} / \mathrm{ha}$ con rendimiento de $3834 \mathrm{t} / \mathrm{ha}$, un vigor de $82 \%$ y una germinación de $89,83 \%$.

Palabras clave: Forraje, manejo agronómico, densidad de población, vigor y germinación.

\begin{abstract}
Data and planting density on the production and quality seed the triticale (X Triticosecale Wittmack). The objective of this study was to evaluate the effects of sowing dates and seeding rates on triticale seed yield and quality. This work was performed during the autumn-winter season in 2006-2007, at the research station of The Technological Institute of Roque, located at $\mathrm{km} 8$ of the highway CelayaJuventino Rosas, Celaya, Guanajuato, Mexico. We used three cultivars of triticale of different genetic origin (Roque I, AN31 and AN-105) with one formula per hectare of 120-100100 , NPK. Three sowing dates were evaluated, November 9, November 23, and December 7, and five seeding rates at each planting date, $50 \mathrm{~kg} / \mathrm{ha}, 75 \mathrm{~kg} / \mathrm{ha}, 100 \mathrm{~kg} / \mathrm{ha}, 125$ $\mathrm{kg} / \mathrm{ha}$ and $150 \mathrm{~kg} / \mathrm{ha}$. The variables evaluated were plant height, ear length, seed yield, vigor, and germination. The experimental design was a randomized complete block design with a factorial arrangement. The optimal sowing date for seed production was November 9, resulting in a yield of 5,305 t/ha. This treatment resulted in the highest values for ear length, plant height, vigor, and germination. When selecting important traits to produce high quality seed, through sowing density, the highest plant population was obtained with $125 \mathrm{~kg} / \mathrm{ha}$ resulting in a yield of $3,834 \mathrm{t} / \mathrm{ha}$, vigor of $82.0 \%$ and germination of $89.83 \%$.
\end{abstract}

Key words: Forage, agronomic management, population density, vigor and germination.

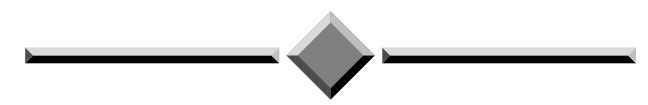

\footnotetext{
Recibido: 20 de septiembre, 2010. Aceptado: 3 de octubre, 2011. Proyecto financiado por la Dirección General de Educación Superior Tecnológica. SEP. México.

2 Instituto Tecnológico de Roque, Celaya, Gto. Km 8 Carretera Celaya-J. Rosas, Celaya. CP. 38110. Tel 01(461)6116362 Ext. 135. Correspondencia: mmendoza66@hotmail.com; elviraciga@yahoo.com.mx; jogurrciga@yahoo.com; arangel_1@yahoo.com; subtec33@yahoo.com. mx; cervan74@yahoo.com.mx
} 


\section{INTRODUCCIÓN}

El triticale es un cereal de invierno, que hoy presenta rendimientos equivalentes o hasta superiores a los del trigo, centeno y avena. El triticale (X Triticosecale Wittmack) se siembra principalmente para consumo animal, especialmente como grano para concentrados, compitiendo exitosamente por calidad y precio con el maíz (Zea mays L.) y cebada (Hordeum vulgare L.) (Rojas 2004).

Actualmente, el triticale constituye una alternativa de producción por la resistencia del centeno y el potencial de rendimiento y las cualidades nutritivas del trigo. El centeno ha aportado al triticale una gran resistencia al frío y a las enfermedades. El triticale es hoy equivalente al trigo en condiciones normales de producción. En condiciones de estrés (temporal), presenta incluso un mejor comportamiento que el trigo: en suelo seco, permite obtener un rendimiento superior; asimismo, en suelo hidromorfo, gracias a su fuerte capacidad para rebrotar, compensará más fácilmente las pérdidas de brotes que un trigo o una cebada (Mendoza et al. 2006).

Entre las características que resaltan es la alta producción de materia seca y la menor pérdida de calidad que presenta con el avance de su fenología, en comparación con el cultivo de avena (Avena sativa L.) y cebada (Romero et al. 1999).

El triticale es una especie difundida prácticamente por todo el mundo, pero en muchos países está todavía en fase de introducción y expansión. Existen programas de mejora de triticale, tanto privado como públicos $y$, en general, hay una cierta correspondencia entre la superficie de triticale cultivada en un país y la intensidad de la mejora que se lleva a cabo en el mismo (Royo 1992). Aún, después de varios años de estudio en esta especie, es muy escasa la información referente a la calidad física y fisiológica de la semilla. En México existen pocas variedades a nivel comercial, y no existen reportes sobre el paquete tecnológico para la producción de semilla de triticale.

La calidad de las semillas se compone de atributos genéticos, físicos, fisiológicos y sanitarios, mismos que se determinan durante el ciclo biológico de la planta y es afectado por factores climáticos como la temperatura, el fotoperiodo y la disponibilidad de humedad, y factores fisiológicos como la nutrición y la competencia entre plantas (Moreno 1996).

Entre las características de calidad fisiológica, la germinación, es la más aceptada para definir la utilidad de la semilla para siembra y la prueba de germinación estándar, la más empleada. Los procedimientos estandar para evaluar la calidad están reglamentados por la International Seed Testing Association (ISTA) y corroborada en México por el Servicio Nacional de Inspección y Certificación de Semillas (SNICS). Algunos autores, mencionan que esta característica es inadecuada para medir la respuesta de la semilla en condiciones de campo, ya que para emerger una plántula requiere de fuerza y uniformidad, característica que no es obtenida por la prueba de germinación estándar o la de viabilidad (Cortez 2000).

La germinación se define como la emergencia y desarrollo de aquellas estructuras esenciales que provienen del embrión y que manifiestan la capacidad de la semilla para producir una planta normal bajo condiciones favorables. La prueba de germinación estándar permite hacer comparaciones del poder germinativo entre diferentes lotes de semilla (Moreno 1996).

Los factores que causan cambios en el nivel de vigor de la semilla incluyen la constitución genética de ésta, el medio ambiente y nutrición de la planta madre, integridad mecánica, deterioro, envejecimiento y patógenos (Perry 1988). El vigor de la semilla comprende aquellas propiedades que determinan el potencial para una rápida y uniforme emergencia y desarrollo de plántulas normales bajo un amplio rango de condiciones de campo. Esta versión toma el resultado de vigor de la semilla y lo cuantifica en términos de una rápida y uniforme emergencia y desarrollo de plántulas normales (Delouche 2002). Una reducción en el vigor de las semillas produce una baja y heterogénea emergencia de plántulas, menor crecimiento y reducción en la producción (Rodríguez y McDonald 1989). El vigor de la semilla es el factor más importante para la calidad, por estar relacionada con una germinación más rápida y uniforme y plántulas más vigorosas y competitivas; se sabe que ésta característica se refleja en el rendimiento (AOSA 1992).

El objetivo de este trabajo fue determinar el efecto de la densidad y la fecha de siembra en el vigor, la germinación y el rendimiento para la producción de semilla de triticale. 


\section{MATERIALES Y MÉTODOS}

El presente trabajo se realizó durante el año 2006 en el ciclo agrícola otoño invierno en el campo experimental de la División de Estudios de Posgrado e Investigación del Instituto Tecnológico de Roque, ubicado en el Km. 8 carretera Celaya-Juventino Rosas, Roque, Celaya, Guanajuato; cuyas coordenadas son $20^{\circ} 32^{\prime}$ de Latitud Norte y $100^{\circ} 45^{\prime}$ de Latitud Oeste, a una altitud de $1762 \mathrm{msnm}$. De acuerdo con la clasificación climática de Koeppen Modificado por García (1998) se hacen las siguientes descripciones: en la región Celaya, los climas predominantes son semi-cálidos, sub-húmedos BS, y C (Wo) con temperaturas medias que van desde $14^{\circ}$ hasta $22^{\circ} \mathrm{C}$ y las precipitaciones varían desde 600 hasta $1000 \mathrm{~mm}$ anuales. Las heladas se inician en los últimos días de septiembre y se establecen de manera franca en octubre, pero en ocasiones se presentan en abril. Los suelos que predominan en la región son los Vertisoles Pélicos y Vertisoles Crómicos, con predominancia de los primeros; estos son de textura arcillosa, planos, de reacción ligeramente alcalina, sumamente fértiles y aptos para una gran variedad de cultivos.

La preparación del terreno consistió en un barbecho, dos pasos de rastra, nivelación y surcado con una separación entre surcos de $0,75 \mathrm{~m}$, la primera siembra del experimento se realizó el 9 de noviembre del 2006, se sembró de forma manual depositando las siguientes cantidades de semilla: $50,75,100,125$ y $150 \mathrm{~kg} / \mathrm{ha}$. La segunda siembra se realizó el 23 de noviembre del 2006 y la tercera el día 7 de diciembre del mismo año, empleando la metodología anterior y con las mismas densidades de siembra. Se fertilizó con la fórmula 120, 100,100 por hectárea, nitrógeno, fosforo y potasio, respectivamente; dividida en dos aplicaciones, en la que se utilizó urea como fertilizante nitrogenado; superfosfato triple como fuente de fosforo y sulfato de potasio como fuente de potasio.

La primera fertilización fue el día de la siembra y la segunda en la fase fenológica de macollamiento. Se realizaron dos deshierbes manuales con hoces de raspa en las hileras de las plantas y parte inferior de los surcos a los 60 y 120 días después de la siembra.

Para este estudio se utilizaron tres cultivares de triticale de diferente origen genético (Roque I, AN-31 y AN-105). El germoplasma empleado es originario del CIMMYT. La variedad Roque I se obtuvo en el Instituto Tecnológico de Roque y las variedades AN31 (hábito invernal, son cultivares de ciclo vegetativo tardío, de varios cortes y para pastoreo) y AN-105 (hábito intermedio, son genotipos de ciclo vegetativo intermedio y cortes múltiples), son triticales apropiados para uso forrajero, generados por la Universidad Autónoma Agraria Antonio Narro (Lozano-del Río et al. 2009). Los genotipos de triticale seleccionados de CIMMYT y de la UAAAN tienen características de grano para la panificación, son erectos de tallos gruesos y resistentes, de tipo amacollado, la edad de cosecha es de aproximadamente 120 días cuando se siembra en noviembre, acortándose el ciclo en siembras a fines de diciembre que a la vez también reduce el rendimiento.

En el transcurso del ciclo vegetativo se eliminaron las plantas fuera de tipo, plantas de malezas y enfermas, que permite corregir problemas de pureza genética que, generalmente, se realizan en los cultivos de granos pequeños, de acuerdo a las normas de certificación del SNICS para producción de semilla (SNICS 2011). En nuestro caso, ésta actividad se realizó en dos ocasiones, a los 60 y 120 días después de la siembra y los residuos se retiraron del lote de producción.

\section{Medición de las variables de campo}

Para la altura de planta, se tomó una lectura con cinta métrica desde la base de la planta hasta la punta de la espiga, tomando 40 muestras al azar de cada surco, posteriormente se obtuvo el promedio como dato representativo de cada unidad experimental, esto se hizo respectivamente para cada tratamiento.

La longitud de espiga consistió en medir con una regla, 40 espigas al azar de cada tratamiento y repetición, posteriormente se obtuvo el promedio de cada unidad experimental.

La cosecha se realizó manualmente, se cortaron las espigas con una hoz de raspa y se envasaron en costales de $50 \mathrm{~kg}$, cada uno marcado con sus respectivos tratamientos, posteriormente se trasladaron al laboratorio de análisis de semillas donde se colocaron en el asoleadero y tres días después se logró el secado final para realizar la trilla. Para estimar el rendimiento se utilizó como parcela útil los dos surcos centrales de cada una. 
Para las variables de calidad fisiológica, se utilizó la semilla cosechada, a la que se determinó el vigor mediante el porcentaje de plántulas normales observadas en el recuento (cuatro días) de la prueba de germinación estándar, en cuatro repeticiones de veinticinco semillas por tratamiento.

La germinación se determinó mediante la prueba estándar, en una cámara de germinación, a una temperatura de $25^{\circ} \mathrm{C}$, el conteo se realizó a los ocho días de la siembra, de acuerdo a la metodología que prescriben las normas establecidas para el análisis de semillas (Moreno 1996).

Se aplicó un diseño experimental de bloques completos al azar con arreglo factorial, se establecieron cinco densidades de siembra con cuatro repeticiones, cuatro surcos de cuatro de longitud, separados a 0,75 $\mathrm{m}$, constituyendo un área de $12 \mathrm{~m}^{2}$. El factor A correspondió a las densidades de siembra y el factor B a las fechas de siembra. Para las pruebas de laboratorio se utilizó un diseño completamente al azar con arreglo factorial. Para ambas, la comparación de medias fue por Duncan $(\mathrm{P} \geq 0,05)$ utilizando el paquete estadístico SAS v.9.0 (2008).

\section{RESULTADOS Y DISCUSIÓN}

En el análisis de varianza se observó que la variable longitud de espiga en la fuente de variación "fecha de siembra" (Factor B) fue fuertemente afectada por el clima, ya que se encontró diferencia altamente estadística al uno porciento de probabilidad. Por ser un componente primario y que influye directamente en el rendimiento, es necesario hacer un mayor número de evaluaciones para definir con exactitud cuál es la mejor fecha de siembra para la producción de semilla. Por otro lado, la fuente de variación "densidad de siembra" (Factor A) y la interacción fecha con densidad de siembra (A x B) no afectaron esta variable, los resultados fueron estadísticamente no significativos. Para esta característica el coeficiente de variación fue de $6,98 \%$, el cual se considera confiable.

Para altura de planta se encontró que todas las fuentes de variación (densidad de siembra, fecha de siembra e interacción) influyeron para que al menos uno de los tratamientos presentara una respuesta diferencial, es decir, se determinaron diferencias estadísticas altamente significativas al 1\%. Para esta variable se reporta un coeficiente de variación de $8,15 \%$. El rendimiento de semilla, también mostró diferencias estadísticas para todas las fuentes de variación a excepción de las repeticiones. Esta respuesta se atribuye principalmente al número de semillas por unidad, asimismo, a las condiciones climáticas, principalmente de temperatura, humedad y fotoperíodo, el coeficiente para esta variable fue de $10,90 \%$. Para el componente fisiológico, que se refiere al vigor y germinación, al igual que para rendimiento hubo diferencias estadísticas altamente significativas para todos los factores, es decir, es necesario analizar puntualmente estas variables ya que definen parte del buen establecimiento del cultivo y los datos demuestran que para obtener una semilla de alta calidad es importante considerar la fecha y densidad de siembra óptima. Para ambas variables resultaron coeficientes de variación de 2,63 y $2,59 \%$, respectivamente.

En el Cuadro 1, se presentan los resultados obtenidos para las diferentes fechas de siembra, se observa que la longitud de espiga, altura de planta, rendimiento de semilla, vigor y germinación fue superior en la siembra realizada el día 9 de noviembre; por ejemplo, en la longitud de espiga existió una diferencia de 1,87 $\mathrm{cm}$ con respecto al peor tratamiento (Fecha de siembra del 7 de diciembre). En este caso, se presentaron tres grupos estadísticos y se aprecia que esta variable influyó fuertemente en el rendimiento de semilla. En la altura de planta se encontró una respuesta similar estadísticamente entre la fecha del 9 y el 23 de noviembre, existiendo una superioridad del tratamiento uno de $36,35 \mathrm{~cm}$ con respecto al tratamiento tres. Para la característica vigor de la semilla se determinó que en la fecha de siembra sí influyeron las características climáticas, y dado que no se encontró diferencia estadística en la fuente de repeticiones; se asume que las propiedades edáficas no afectaron esta variable. De acuerdo con los resultados, la mejor fecha de siembra para vigor fue la del 9 de noviembre con un valor de $85,20 \%$ diferente estadísticamente a las otras fechas de siembra, a medida que se retrasó la siembra el vigor se vio mas afectado. Entre la mejor fecha de siembra y la realizada en el mes de diciembre se observó una diferencia de 10,45 , esto significa que para obtener una semilla de alta calidad en vigor es necesario considerar los factores de clima, que de acuerdo con estos datos se muestra una tendencia negativa bastante pronunciada que llega a tener un valor de 74,75. Otra variable 
Cuadro 1. Respuesta del cultivo de triticale a diferentes fechas de siembra en el ciclo otoño invierno del año 2006-2007, en Celaya, Gto., México.

\begin{tabular}{lccccc}
\hline Fechas de siembras & $\begin{array}{c}\text { Longitud de } \\
\text { espiga }(\mathbf{c m})\end{array}$ & $\begin{array}{c}\text { Altura de planta } \\
(\mathbf{c m})\end{array}$ & $\begin{array}{c}\text { Rendimiento se- } \\
\text { milla (t/ha) }\end{array}$ & $\begin{array}{c}\text { Vigor } \\
(\boldsymbol{\%})\end{array}$ & $\begin{array}{c}\text { Germinación } \\
(\boldsymbol{\%})\end{array}$ \\
\hline 9 de noviembre & $13,47 \mathrm{a}$ & $104,47 \mathrm{a}$ & $5,305 \mathrm{a}$ & $85,20 \mathrm{a}$ & $93,10 \mathrm{a}$ \\
23 de noviembre & $12,87 \mathrm{~b}$ & $102,42 \mathrm{a}$ & $3,670 \mathrm{~b}$ & $82,10 \mathrm{~b}$ & $89,55 \mathrm{~b}$ \\
7 de diciembre & $11,60 \mathrm{c}$ & $68,12 \mathrm{~b}$ & $2,673 \mathrm{c}$ & $74,75 \mathrm{c}$ & $83,05 \mathrm{c}$ \\
\hline LSD $(5 \%)$ & 0,045 & 0,090 & 0,260 & 1,35 & 1,46 \\
\hline
\end{tabular}

Letras similares significa que no existe diferencia estadística, letra distinta indican diferencia entre tratamientos (Duncan 5\%). LSD (Diferencia mínima significativa).

que define la calidad fisiológica de la semilla y con ello el agricultor asegura un buen establecimiento del cultivo en campo es la germinación; también existieron diferencias estadísticas entre fechas de siembra. De acuerdo a la prueba de comparación de medias, se formaron tres grupos estadísticamente diferentes, se aprecia el mismo comportamiento lineal, es decir, a medida que se realizan en forma más tardía las fechas de siembra, la germinación se redujo, la primer fecha arrojó el 93,10\% de semilla germinada, la segunda el 89,55 y la última fecha de siembra presentó el 83,05\% (Cuadro 1). Estos resultados se atribuyen a que la fecha de siembra tres es considerada como la más tardía, las condiciones principalmente de temperaturas bajas dañan el llenado de la semilla afectando de esta manera la calidad fisiológica de la misma.

Con respecto al otro factor de estudio, densidad de siembra, se encontró que para longitud de espiga el mejor tratamiento fue $150 \mathrm{~kg} / \mathrm{ha}$ con valor de $13 \mathrm{~cm}$ de longitud, aunque se observaron diferencias estadísticas entre densidades, solo se apreció una diferencia entre el mejor tratamiento $(150 \mathrm{~kg} / \mathrm{ha})$ y los tratamientos más bajos de $0,67 \mathrm{~cm}$ aproximadamente con las densidades de 50 y $75 \mathrm{~kg} / \mathrm{ha}$ (Cuadro 2).

Para altura de planta, se observan dos grupos estadísticamente diferentes; se detectó que a medida que la densidad de siembra aumentó la altura de planta se incrementó de 83 a $101,9 \mathrm{~cm}$, la altura de planta se afectó positivamente, de tal manera, que las mejores densidades de siembra se obtuvieron cuando se establecieron parcelas con 125 y $150 \mathrm{~kg} / \mathrm{ha}$ con valores de 96,4 y $101,9 \mathrm{~cm}$, respectivamente; los genotipos utilizados en el ensayo mostraron variaciones entre el
18 y $24,7 \%$, en esta característica (Cuadro 2). Dado que el cultivo de triticale, en la actualidad, su mayor destino es para forraje y la altura de planta juega un papel importante en el rendimiento, por lo que conjuntamente a la calidad nutritiva, la cual en la etapa de encañe-embuche (inicio de espiga y llenado de grano), es similar a la alfalfa, resulta una buena alternativa para las explotaciones agropecuarias (Diannelis et al. 1995, Bejar et al. 2004, Collar y Aksland 2001).

En el rendimiento de semilla, la mejor densidad de siembra fue la de $150 \mathrm{~kg} / \mathrm{ha}(4,7 \mathrm{t} / \mathrm{ha})$ sobresaliente estadísticamente a los otros cuatro tratamientos y con una superioridad de $854 \mathrm{~kg} / \mathrm{ha}$ con respecto a la densidad de $50 \mathrm{~kg} / \mathrm{ha}$. Es importante señalar, que entre 50, $75 \mathrm{y}$ $100 \mathrm{~kg} / \mathrm{ha}$ no se aprecia diferencia estadística, pues la diferencia entre ellos fue muy pequeña. Los costos de producción de semilla se vieron afectados por la cantidad de semilla sembrada; estos resultados conllevan al uso racional de la semilla utilizada por el agricultor o empresa de semilla dedicada a este rubro. Por otro lado, considerando que los mejores rendimientos se obtuvieron con la densidad más alta, se establece la hipótesis de que puede ser rentable incrementar la densidad de siembra (Cuadro 2). Rivera et al. (2008) concluyeron que el rendimiento de semilla en avena fue mayor en las densidades de 80 y $100 \mathrm{~kg} / \mathrm{ha}$ en comparación con 60 y $120 \mathrm{~kg} / \mathrm{ha}$. Otro reporte similar lo realizaron Vela (1997) y Gómez et al. (2001) para el cultivo de cebada.

La calidad fisiológica (vigor y germinación) de la semilla se vio afectada por la cantidad de semilla a establecer en campo (densidad de siembra) (Cuadro 2), primeramente la variable vigor mostró cuatro grupos estadísticos diferentes, los mejores resultados 
Cuadro 2. Efecto de las densidades de siembra en caracteres agronómicos de triticale en el ciclo otoño invierno del año 2006 en Celaya, Gto., México.

\begin{tabular}{ccccccc}
\hline $\begin{array}{c}\text { Densidad de } \\
\text { siembra (kg/ha) }\end{array}$ & $\begin{array}{c}\text { Longitud de } \\
\text { espiga (cm) }\end{array}$ & $\begin{array}{c}\text { Altura de planta } \\
(\mathbf{c m})\end{array}$ & $\begin{array}{c}\text { Rendimiento } \\
\text { semilla (t/ha) }\end{array}$ & Vigor (\%) & Germinación (\%) \\
\hline 50 & $12,41 \mathrm{~b}$ & $83,0 \mathrm{~b}$ & $3,81 \mathrm{~b}$ & $79,83 \mathrm{c}$ & $87,92 \mathrm{~b}$ \\
75 & $12,33 \mathrm{~b}$ & $87,7 \mathrm{~b}$ & $4,00 \mathrm{~b}$ & $79,58 \mathrm{c}$ & $87,50 \mathrm{~b}$ \\
100 & $12,75 \mathrm{ab}$ & $89,2 \mathrm{~b}$ & $3,89 \mathrm{~b}$ & $81,75 \mathrm{ab}$ & $90,42 \mathrm{a}$ \\
125 & $12,75 \mathrm{ab}$ & $96,4 \mathrm{a}$ & $3,83 \mathrm{~b}$ & $82,00 \mathrm{a}$ & $89,83 \mathrm{a}$ \\
150 & $13,00 \mathrm{a}$ & $101,9 \mathrm{a}$ & $4,67 \mathrm{a}$ & $80,17 \mathrm{bc}$ & $87,17 \mathrm{~b}$ \\
\hline LSD (5\%) & 0,060 & 0,116 & 0,35 & 1,75 & & 1,89 \\
\hline
\end{tabular}

Letras similares, significa que son estadísticamente iguales. (Duncan 5\%), LSD (Diferencia mínima significativa).

se presentaron cuando la siembra se realizó con 125 $\mathrm{kg} / \mathrm{ha}$ con un valor de vigor de $82 \%$, caso contrario, sucedió cuando se usaron 50 y $75 \mathrm{~kg} / \mathrm{ha}$. Casi el mismo comportamiento se aprecia cuando se trabaja con la germinación de la semilla, los mejores resultados se encontraron con 100 y $125 \mathrm{~kg} / \mathrm{ha}$ estadísticamente similares con valores de 90,42 y $89,93 \%$, respectivamente. Al respecto, Rivera et al. (2008) concluyeron que la densidad de siembra de $40 \mathrm{~kg} / \mathrm{ha}$ fue mejor para vigor y germinación en avena. Esto confirma que el exceso de población debilita las plantas afectando la acumulación de reservas nutritivas que repercuten en la calidad fisiológica de la semilla.

En las medias del rendimiento y variables de la calidad fisiológica (Cuadro 3), se aprecia que la mejor interacción se tuvo con la fecha de siembra realizada el 9 de noviembre y la densidad más alta que fue la de 150 $\mathrm{kg} / \mathrm{ha}$, aunque estadísticamente similar, con la densidad de $125 \mathrm{~kg} / \mathrm{ha}$, con valores de 6,6 y $6,5 \mathrm{t} / \mathrm{ha}$, respectivamente, los rendimientos más bajos se encuentran con la fecha tres ( 7 de diciembre) y la densidad cuatro, estadísticamente similar con la fecha tres y la densidad tres.

A través de densidades y fechas de siembra se encontró que el mejor rendimiento de semilla fue de 6,6 t/ha, la mejor calidad fisiológica se obtuvo cuando se tuvo un vigor de 87,25 y una germinación de $96,25 \%$, esta respuesta se encuentra con la fecha de siembra realizada el 21 de noviembre (fecha dos) y con la densidad de siembra más baja (50 kg/ha), estadísticamente similar en vigor a la fecha dos con la densidad cuatro $(125 \mathrm{~kg} / \mathrm{ha})$ y en la germinación con la fecha dos y la densidad tres $(100 \mathrm{~kg} / \mathrm{ha})$. En algunos estudios
Cuadro 3. Promedios de rendimiento, vigor y germinación entre la interacción de fechas de siembra $(F)$ y densidades de siembra (D) en el cultivo de triticale en el ciclo otoño invierno del año 2006 en Celaya, Gto., México.

\begin{tabular}{cclclll}
\hline F-D* & $\begin{array}{c}\text { Rendimiento } \\
(\mathbf{t} / \mathbf{h a})\end{array}$ & Vigor $(\%)$ & $\begin{array}{c}\text { Germinación } \\
(\%)\end{array}$ \\
\hline $1-1$ & 4,45 & bc & 79,25 & de & 87,25 & cde \\
$1-2$ & 4,85 & b & 79,00 & ef & 88,25 & cd \\
$1-3$ & 4,12 & cd & 83,50 & ab & 94,25 & a \\
$1-4$ & 6,50 & a & 83,00 & c & 89,25 & cd \\
$1-5$ & 6,61 & a & 82,75 & c & 88,75 & cd \\
$2-1$ & 3,05 & g & 87,25 & a & 96,25 & a \\
$2-2$ & 3,46 & efg & 83,75 & bc & 89,50 & cd \\
$2-3$ & 3,84 & def & 85,25 & abc & 96,06 & a \\
$2-4$ & 3,87 & cde & 87,50 & a & 93,50 & ab \\
$2-5$ & 4,14 & cd & 82,25 & cd & 90,25 & bc \\
$3-1$ & 3,94 & cde & 73,00 & g & 80,25 & g \\
$3-2$ & 3,70 & def & 76,00 & fg & 84,75 & ef \\
$3-3$ & 1,34 & h & 73,50 & g & 81,00 & g \\
$3-4$ & 1,14 & h & 75,75 & g & 86,75 & de \\
$3-5$ & 3,25 & efg & 75,50 & g & 82,50 & fg \\
\hline LSD (5\%) & 0,604 & 3,27 & 3,03 & \\
\hline
\end{tabular}

*Fecha de siembra (1): 19 de noviembre, (2): 23 de noviembre y (3) el 7 de diciembre del 2006. Densidad de siembra: (1): $50 \mathrm{~kg} /$ ha, (2). $75 \mathrm{~kg} / \mathrm{ha}$, (3). $100 \mathrm{~kg} / \mathrm{ha}$, (4). $125 \mathrm{~kg} / \mathrm{ha}$, (5). $150 \mathrm{~kg} / \mathrm{ha}$ (Duncan 5\%). LSD (Diferencia mínima significativa). 
realizados por Match et al. (2007) en un experimento en Córdoba, Argentina para rendimiento de grano en triticale a través de selección recurrente bajo condiciones de secano, obtuvieron un rendimiento de 1,45 t/ha en dos ciclos de selección. Vela et al. (1997) y Rivera et al. (1997) reportaron una baja en el rendimiento al incrementar la densidad de siembra en cebada, lo cual puede ser influenciado por el exceso de crecimiento con tallos débiles que generalmente provoca acame del cultivo por menor disponibilidad de luz solar, esto afecta la calidad de la semilla y el rendimiento.

\section{LITERATURA CITADA}

AOSA. 1992. Seed Vigor Testing handbook. Association Official Seed Analists Contribution No. 32 to the handbook of seed testing. US. 6(2):1-126.

Bejar, HM; Ammar, K; Lechuga, ML; Fematt, G; Cano, JM. 2004. El cultivo de triticale como elemento importante en la sustentabilidad de agrosistemas. Centro de Investigación para los Recursos Naturales. Chihuahua, México. Folleto Técnico No. 35 p.

Collar, C; Aksland, G. 2001. Harvest effects on yield and quality of winter forage. Proc. 31st California Alfalfa and Forage Symposium., Ca. U. C. Cooperative Extension. University of California, Davis. Modesto:133-142.

Cortéz, BE. 2000. Calidad de semilla de genotipos de fríjol producidos en dos localidades. Tesis de Maestría UAAAN (Universidad Autónoma Agraria Antonio Narro), Saltillo, Coahuila, México. 83 p.

Delouche, JC. 2002. Germinación, deterioro y vigor de semillas. Seed News. Mississipi State University. EUA. Consultado 15 ago. 2008. Seednews@seednews.inhr

Diannelis, CUY; Arriojas, I; Dávila, C. 1995. Efecto de la fertilización en la asociación de Kikuyo-Alfalfa (Pennisetum clandestinum - Medicago sativa) II. Contenido de proteína y digestibilidad. Mérida, Venezuela. Zootecnia Trop. 13(2):183-198.

García, E. 1998. Modificaciones al sistema de clasificación climática de Köppen para adaptarlos a las condiciones de la República Mexicana.UNAM (Universidad Nacional Autónoma de México). México. 246 p.

Gómez, MR; Turrent, FA; Ortiz, C; Peña, OB. 2001. Productividad de cebada maltera. II. Análisis de las Interacciones de seis factores de la producción. Agricultura Técnica en México 27(2):95-105.

Lozano-del Río AJ; Zamora-Villa, VM; Ibarra-Jiménez, L; Rodríguez-Herrera, SA; de la Cruz-Lázaro, E; de la
Rosa-Ibarra, M. 2009. Análisis de la interacción genotipo ambiente mediante el modelo AMMI y potencial de producción de triticales forrajeros (X Triticosecale Wittm.) Universidad y Ciencia, México. 25(31):81-92.

Match, R; Manero de Zumelzu, D; Davidenco, V. 2007. Selección recurrente en triticale hexaploide (X Triticosecale Wittmack) bajo condiciones de secano. Agriscientia (Córdoba) 24(1):57-60.

Mendoza, M; Andrio, E; García, JG. 2006. El Triticale: un cereal para forraje con futuro para zonas marginadas. Artículo de Difusión. Revista TECNOAGRO, México. 26:48-50.

Moreno, CP. 1996. Análisis físico y biológico de semillas agrícolas UNAM-FAO (Universidad Nacional Autónoma de México-Organización de las Naciones Unidas para la Agricultura y la Alimentación). México, Distrito Federal. 393 p.

Perry, DA. 1988. El concepto de vigor de la semilla y su relevancia con respecto a las técnicas de producción de semilla. Producción moderna de semillas. Tomo 11. Edit. Agropecuaria, hemisferio sur. S.R. L. Montevideo, Uruguay. p. 693-701.

Rivera, RJG; Cortez, BE; Urbina, AR; Andrio, EE. 1997. Componentes de rendimiento de trigo (Triticum aestivum.L.) variedad Salamanca S75. In Vll congreso nacional y desarrollo Tecnológico Agropecuario. DGETA (Dirección General de Educación Tecnológica Agropecuaria). Morelia, Michoacán. p. 106.

Rivera, RJG; Cortez, BE; Peraza, LFA; Serratos, AJC; Posos, PP; Guevara, GR; Torres, PI; Guzmán, MSH. 2008. Agronomic trials associated to yield and quality in oat seeds. Asian Journal of Plant Sciences 7(8):767-770.

Rojas, GC. 2004. Manual de producción de bovinos de carne para la VIll, 1X y X regiones, INIA, Corillanca, Chile. $25 \mathrm{p}$.

Rodríguez, A; McDonald, MB. 1989. Seed quality influence on plant grow and dinitrogen fixation on red field bean. Crop science 29:1309-1314.

Romero, O; Rojas, C; Butendieck, N; Hazard, S. 1999. Producción de materia seca y calidad nutritiva de tres especies de cereales: avena, cebada y triticale para ensilaje. XXXIV Reunión Anual de la Sociedad Chilena de Producción Animal (SOCHIPA), Temuco, Chile. 27-29 de octubre. Sociedad Chilena de Producción Animal, Santiago Chile. p. 49-50.

Royo, C. 1992. El triticale. Base para el cultivo y aprovechamiento. Madrid, España. Agro-vías. Mundi-prensa. 96 p.

SAS, 2002. Statistical Analysis System. Version 9.0 Institute Inc. Cary NC, USA. 
SNICS. 2011. Servicio Nacional de Inspección y Certificación de Semillas. www.snics.gob.mx

Vela, LB. 1997. Rendimiento y calidad de semilla de cebada (Hordeum vulgare L) en respuesta a densidad de siembra y fertilización. Tesis de Maestría. Instituto Tecnológico Agropecuario No. 33. Roque, Celaya, Gto., México. 86 p.
Vela, LB; Andrio, EE; Rivera, RJG; Rangel, LJA. 1997. Rendimiento y calidad de semilla de cebada (Hordeum vulgare L.). Respuesta a la densidad de siembra y fertilización. Vll Congreso Nacional de Investigación y Desarrollo Tecnológico Agropecuario, DEGTA (Dirección General de Educación Tecnológica Agropecuaria). Celaya; Gto., México. p. 147. 